\title{
In situ silver nanoparticle synthesis on 3D-printed polylactic acid scaffolds for biomedical applications
}

\author{
Semilh Calamalk ${ }^{1, a)}$ (10, Menekse Ermis ${ }^{2}$ \\ ${ }^{1}$ Department of Basic Pharmaceutical Sciences, Faculty of Pharmacy, Hacettepe University, Ankara 06100, Turkey \\ ${ }^{2}$ BIOMATEN, Middle East Technical University (METU) Center of Excellence in Biomaterials and Tissue Engineering, Ankara 06800, Turkey

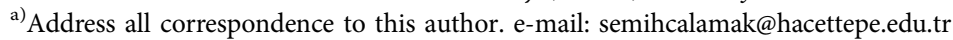

Received: 25 May 2020; accepted: 27 July 2020

An ultraviolet (UV) irradiation-based in situ silver nanoparticle (AgNP) synthesis approach has drawn significant attention for functionalizing a great variety of biomaterials. Here, we designed an AgNP-functionalized 3Dprinted polylactic acid (PLA) composite scaffold with a green physical approach by employing the UV irradiation $(1,2$ and $3 \mathrm{~h})$ method without using any reducing agent or heat treatments. In situ AgNP synthesis was performed under different UV exposure times. The zeta sizer analysis results demonstrated that AgNPs were highly monodisperse with the particle size of $20 \pm 2.2,30 \pm 3.6$, and $50 \pm 4.8 \mathrm{~nm}$ under various UV light exposure times. In situ synthesis of AgNPs on 3D-printed PLA scaffolds significantly changed the surface hydrophilicity of the 3D-printed scaffolds. These results showed that UV irradiation-based in situ AgNP synthesis on 3D-printed PLA scaffolds can be useful in various biomedical applications, such as cell culture scaffolds, biosensors, and wound healing applications.

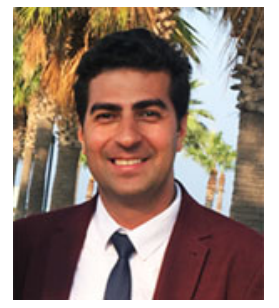

\begin{abstract}
Dr. Semih Calamak is currently working as a Research Assistant at the Department of Basic Pharmaceutical Sciences, Faculty of Pharmacy at Hacettepe University. In 2010, he completed double major undergraduate degrees in Biology and Chemistry at Gazi University. He obtained his M.Sc. and Ph.D. degrees in Nanotechnology and Nanomedicine in 2012 and 2018, respectively, from Hacettepe University. During his doctoral studies, he was appointed as a visiting scientist at Stanford University, Canary Cancer Early Detection Center between December 2014 and December 2015. His research interests include the applications of nano-/microscale technologies and biomaterials in regenerative medicine, and specifically focus on the nano-/micro-patterned biomaterials and their tissue engineering applications, 3D-bioprinted tissue scaffolds, and microfluidic systems. Dr. Calamak has authored and co-authored 21 published SCI indexed journal papers in esteemed journals such as PNAS, Advanced Science, Small, Drug Delivery and Advanced Biosystems. His scientific works have been highlighted and recognized by international organizations including Fox News (USA), Genetic Engineering and Biotechnology News, and Optics \& Photonics News. He has 30 international conference proceeding papers. Moreover, he has authored three book chapters and applied for three patents with his collaborators.
\end{abstract}

\section{Introduction}

The surface modification of biomaterials using micro-/nanoparticles has been gaining popularity to improve materials properties, including hydrophilicity, surface-free energy, and roughness, without affecting the materials thermal and mechanical properties. For surface decoration of the biomaterials, silver nanoparticles (AgNPs) have attracted considerable interest due to their potentials in various applications such as antimicrobial activity [1], sensing [2], therapeutic activity [3], and catalytic activity [4]. In the literature, many methods have been used for the synthesis of AgNPs. The chemical reduction approach is the most commonly used for the reduction of $\mathrm{Ag}^{+}$ions to synthesize nanoparticles [5]. In the chemical reduction approach, sodium borohydride [6], hydrazine [7], N,N-dimethylformamide [8], and other organic compounds are used as a reducing agent. However, these kinds of chemical reducing agents not only cause environmental toxicity but also have biological risks. In this regard, there is an 
urgent need for a low-cost and environmentally green approach for AgNP synthesis.

In the literature, AgNPs have been synthesized using a variety of photoreduction methods, including laser [9], microwave [10], ultrasonic waves [9], microwave [10], ultrasonic wave [11], and gamma [12] and ultraviolet (UV) $[13,14]$ process in aqueous solution and silver salts. Recently, the UV irradiationmediated AgNP synthesis approach has become more attractive due to its excellent properties such as energy efficiency, costeffectiveness, and environmentally friendliness [15, 16]. In our previous works, AgNPs were in situ synthesized in electrospun fibroin nanofibers by UV reduction of $\mathrm{Ag}^{+}$ions. Also, release kinetics, cytotoxicity, and antimicrobial activity of the synthesized AgNPs were elaborately investigated [13, 14]. In another study, Son et al. utilized a UV irradiation approach to produce nanofibers decorated by AgNPs. In this study, various stabilizing agents such as citric acid and carboxymethyl cellulose were used [17]. In the literature, it was shown that the UV irradiation method allows the in situ AgNP synthesis on various materials, including films [18], fibers [14], and ceramics [19]. Also, in situ UV irradiation synthesis of AgNPs on the various materials has been considered an ideal, nontoxic, and low-cost approach [20, 21].

PLA is generally used in orthopedics and dental applications. Also, PLA-based materials showed great application potential as patient-specific surgical implants, including screws, pins, and sutures due to its excellent mechanical properties. To enhance bone and PLA integration, PLA-based materials are mainly coated with an inorganic material such as tricalcium phosphate, hydroxyapatite, and AgNPs [22]. It has been reported that the surface modification of PLA-based materials improves cell biocompatibility of the biomaterial without changing mechanical biocompatibility [22, 23, 24]. Also, PLA is immunologically inert and biodegradable (6 months to 2 years). These properties especially make it an attractive material for use in the medical field [25]. However, there are still some limitations to using PLA as a cell scaffold, such as hydrophobicity and cell adhesion difficulty, limiting clinical use [26, 27]. In the literature, there are various surface functionalization approaches to adjust the surface properties of PLA. These approaches are surface hydrolysis, surface polymerization, and plasma treatment $[28,29,30]$. These surface modifications mainly generate surface roughness and increase surface hydrophilicity, which is crucial for cell adhesion. In a study, Kao et al. utilized polydopamine for surface functionalization of 3D-printed PLA via deep coating into a dilute dopamine solution at $\mathrm{pH}$ 8.5. The polydopamine surface modification was used to provide mussel-inspired surfaces to increase cell attachment and differentiation of human adipose-derived stem cells [26]. In another study, $\mathrm{Li}$ et al. produced gelatin/nanohydroxyapatite and ponericin-grafted $3 \mathrm{D}$-printed
PLA meshes following dopamine modification on the 3D-printed PLA. The results showed that dopamine modification increased the hydrophilicity of the PLA scaffolds. In addition, gelatin/nanohydroxyapatite- and ponericin-coated 3D-printed PLA meshes enhanced the alkaline phosphatase (ALP) secretion [27]. Teixeira et al. fabricated 3D-printed PLA scaffolds with a porous structure with increased mechanical behaviors to mimic the bone structure. To achieve that, they carried out the polydopamine-activated type I collagen functionalization on the $3 \mathrm{D}$-printed samples to increase the scaffolds' bioactivity. The results demonstrated that porcine bone marrow stem cells, which were cultured on type I collagen-modified 3D-printed scaffolds, produced higher ALP after 21-day culture [23].

This research is focused on 3D-printed PLA scaffolds and innovative in-situ AgNP synthesis on these materials as surface functionalization. In our previous work, we used a UV irradiation approach to synthesize AgNPs in electrospun nanofibers, which exhibit high biocompatibility and antibacterial activity. Here, we aimed to develop AgNP-functionalized 3D-printed PLA composite materials with an energy and cost-effective UV irradiation method. The proposed in situ synthesis method is environmentally friendly and does not require any reducing agent, which is dangerous for the environment. To the best of our knowledge, this is the first report in which in situ UV irradiation method was used to synthesize AgNPs on 3D-printed scaffolds. Using this approach, we produced AgNP-functionalized 3D-printed PLA scaffolds with various AgNP size and surface hydrophilicity by adjusting UV exposure time.

\section{Results and Discussion}

\section{CAD design and printing parameters of 3D-printed PLA scaffolds}

The creation of 3D-printed PLA scaffolds is based on the development of the CAD models. In this study, we used the Sketchup software to design CAD files in an STL format. 3D-printed PLA scaffolds were designed in orthogonal and tetragonal layer orientation via a layer-by-layer approach with the schematic image shown in Fig. 1(a). The CAD designs of PLA models with orthogonal and tetragonal geometries were constructed, as shown in Figs. 1(b) and 1(c). In the CAD designs, strut diameter of each scaffold was maintained consistently as $0.4 \mathrm{~mm}$ with various strut space and layer orientations. The 3D PLA scaffolds were named according to layer orientations and strut spaces [Figs. 2(a)-2(d)]. The bright-field image was carried out to investigate the interior structures and morphology of the 3D-printed PLA scaffolds, as shown in Fig. 2. 


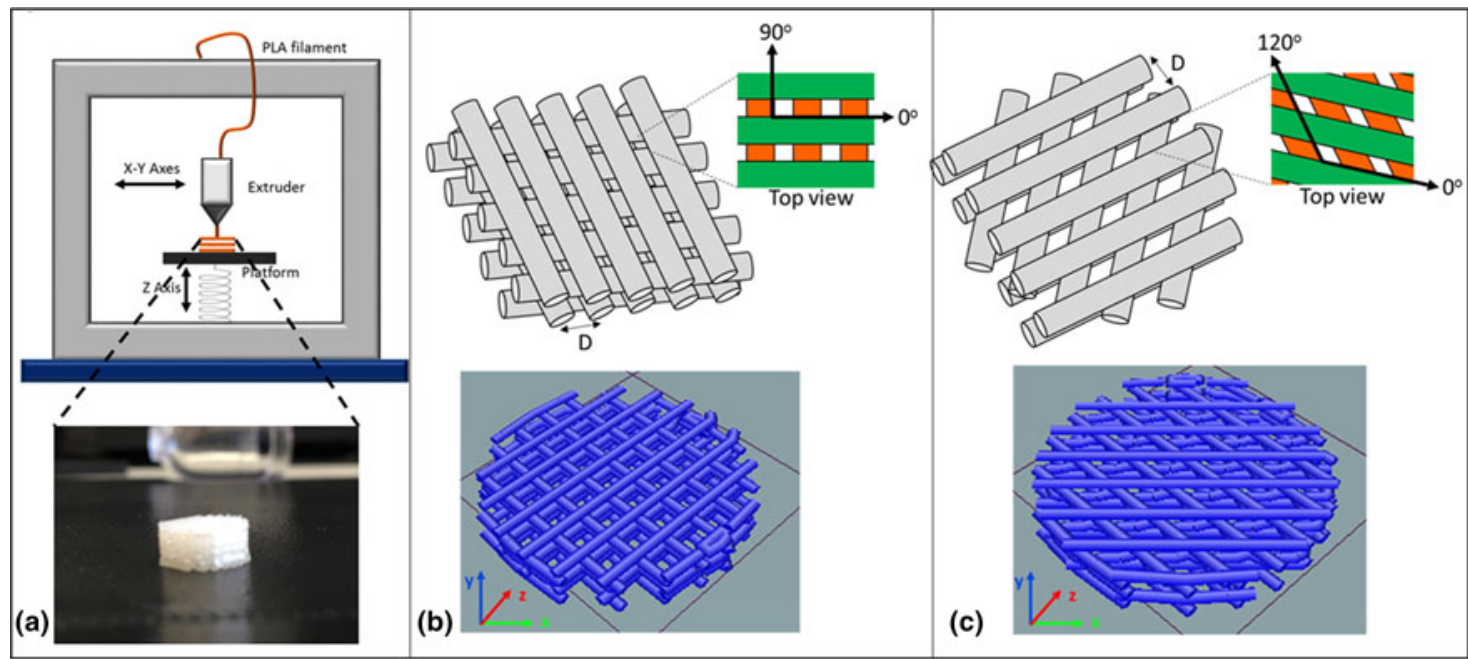

Figure 1: FDM-based 3D printer system and CAD designs of 3D-printed PLA scaffolds. (a) Schematic illustration of FDM-based 3D printer. (b) CAD design of orthogonal 3D scaffolds which have $90^{\circ}$ layer orientation. (c) CAD design of tetragonal 3D scaffolds that have $120^{\circ}$ layer orientation (D: strut space).

\section{Morphology of 3D-printed PLA scaffolds}

The structure of 3D-printed PLA scaffolds was characterized by using bright-field images of the light microscope. Figure 2 demonstrates the macroscopic images of the 3D-printed PLA scaffolds of various mesh sizes and layer orientations. Bright-field and macroscopic images showed that the diameters of the scaffolds were $1 \mathrm{~cm}$. At $4 \times$ magnification, it is clear that the extruded strut diameters of printed scaffolds with different geometries were maintained consistency as $0.4 \mathrm{~mm}$, which were in accordance with the CAD design [Figs. 2(a) and 2(b)]. PLA 90 was chosen for UV-irradiated in situ AgNP synthesis as a model scaffold for the next steps.

\section{In-situ AgNP synthesis and characterization}

The mechanism of the in situ AgNP synthesis with UV irradiation is shown in Fig. 3(a). During the UV irradiation process,
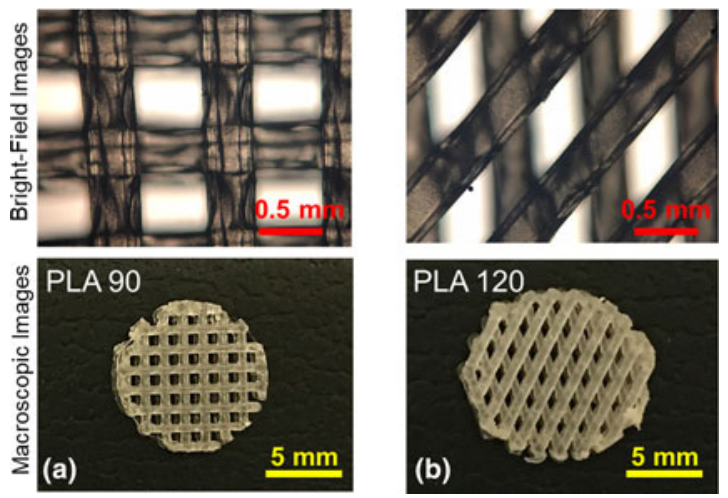

Figure 2: Bright-field and macroscopic images of the 3D-printed PLA scaffolds with orthogonal and tetragonal geometry and $90^{\circ}$ and $120^{\circ}$ layer orientations. (a) PLA 90 (strut space: $0.5 \mathrm{~mm}$ layer orientation: $90^{\circ}$ ) and (b) PLA 120 (strut space: $0.5 \mathrm{~mm}$ layer orientation: $120^{\circ}$ ).
$\mathrm{Ag}^{+}$ions are reduced by $\mathrm{e}_{\mathrm{aq}}^{-}$hydrated electrons, which occur during irradiation in solution. Also, hydrogen atoms formed by the $e_{\mathrm{aq}}^{-}$hydrated electrons in the water solution can also reduce the $\mathrm{Ag}^{+}$ions. The electron capture of the $\mathrm{Ag}^{+}$ions is high; thus, a large number of neutral $\mathrm{Ag}^{0}$ atoms can be produced in the reaction solution. The neutral $\mathrm{Ag}^{0}$ atoms encounter the $\mathrm{Ag}^{+}$ions to form relatively stable $\mathrm{Ag}^{2+}$ species, which leads to the formation of AgNPs [16] [Fig. 3(a)]. The color of the silver precursor solution at various UV exposure time intervals changed from colorless to light brown. Also, the same trend was shown on 3D-printed PLA scaffolds. AgNP absorption and scattering properties can be adjusted by controlling the particle size, shape, and the local refractive index near the particle surface. It has been reported that the optical properties of spherical AgNPs are mainly dependent on the nanoparticle diameter $[31,32,33]$. The colloidal solutions of AgNPs demonstrate various colors due to light absorption and scattering in the visible region based on the plasmon resonance, the frequency at which conduction electrons oscillate in response to the alternating electric field of incident electromagnetic radiation [34, 35]. The AgNP formation was also confirmed by measuring surface plasmon resonance (SPR) peaks. The characteristic of the AgNP SPR peak was recorded to be around $400-460 \mathrm{~nm}$. The absorption peaks of AgNPs were found to show a single sharp peak at 422, 426, and $435 \mathrm{~nm}$ for PLA/Ag(1), PLA/Ag(2), and PLA/Ag(3), respectively [Fig. 1(c)]. The resonance wavelength collected in the experiments exhibited red shifts according to the AgNP particle size. These red shifts and increased absorbance with further UV exposure time demonstrated that the size and concentration of AgNPs had increased because the photoinduced nucleation of AgNPs had enhanced in the solution $[16,36]$. The optical properties of AgNPs significantly change when particle size, 
(a)

Figure 3: In situ AgNP synthesis with the UV irradiation method. (a) Schematic illustration of the in situ AgNP synthesis by using the UV irradiation method, (b) The colorimetric changes of AgNP solutions and $\mathrm{Ag}^{+}$containing (PLA/Ag) 3D-printed scaffolds after various UV exposure times. (c) UV-visible spectra of AgNP solutions after different UV exposure times.

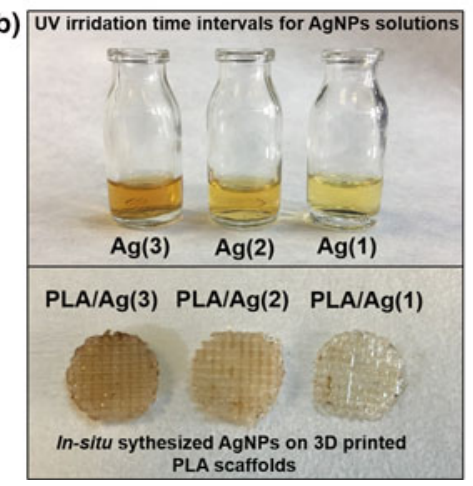

(c)

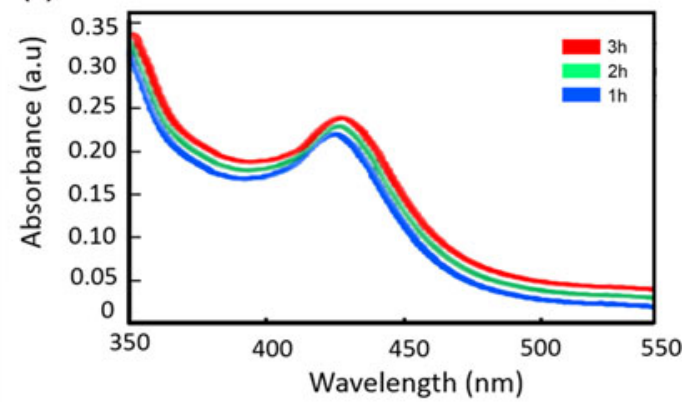

concentration, and aggregation increase in the AgNP solution. In these cases, the conduction electrons near each particle surface become delocalized and are shared among neighboring particles. These electron interactions increased electric field enhancements and resulted in surface resonance shifts to lower energies, causing the absorption and scattering peaks to red-shift to longer wavelengths $[16,36,37]$. The presence of the single SPR peak demonstrated that the synthesized AgNPs were spherically shaped, and these results also were confirmed by transmission electron microscopy (TEM) analysis.

The surface morphology and size distribution of UV lightirradiated AgNPs are shown in Fig. 4. In this approach, $\mathrm{Ag}^{+}$ ions were reduced by UV light irradiation at different time intervals (1, 2, and $3 \mathrm{~h}$ ). A Zeta sizer and TEM were utilized to investigate the sizes and morphologies of the synthesized AgNPs. TEM images showed that the AgNPs solution consists of well-dispersed particles of mainly spherical shape and size, depending on the UV light exposure time. Highly monodisperse AgNPs with particle sizes of $20 \pm 2.2 \mathrm{~nm}$ [Fig. 4(a)], 30 $\pm 3.6 \mathrm{~nm}$ [Fig. 4(b)], and $50 \pm 4.8 \mathrm{~nm}$ [Fig. 4(c)] were synthesized using the in situ UV irradiation method. Furthermore, synthesized AgNPs were highly stable and had zeta potentials of 5.68, 4.37, and $8.32 \mathrm{mV}$ for $\operatorname{Ag}(1), \operatorname{Ag}(2)$, and $\operatorname{Ag}(3)$ solutions, respectively. The size distributions and zeta potential of the AgNPs are summarized in Table 1.

These results confirmed the uniform size distribution of the AgNPs in ultrapure water. Although some particles seem in nonuniform morphology in TEM images, both the Zeta sizer and UV-visible spectroscopy analysis confirmed the excellent colloidal stability of the AgNPs.

In the literature, it has been reported that the size distribution and agglomeration of AgNPs can be adjusted by using stabilizers such as polyvinyl alcohol, polyvinylpyrrolidone, polyethylene glycol, and PLA. Among them, PLA is an ideal material to adjust the size and morphology of the AgNPs. Also, it is able to act as a capping agent with its hydroxyl and carbonyl groups to prevent undesired agglomeration of AgNPs [31]. In this study, we synthesized AgNPs on 3D-printed PLA scaffolds in situ and characterized interactions 

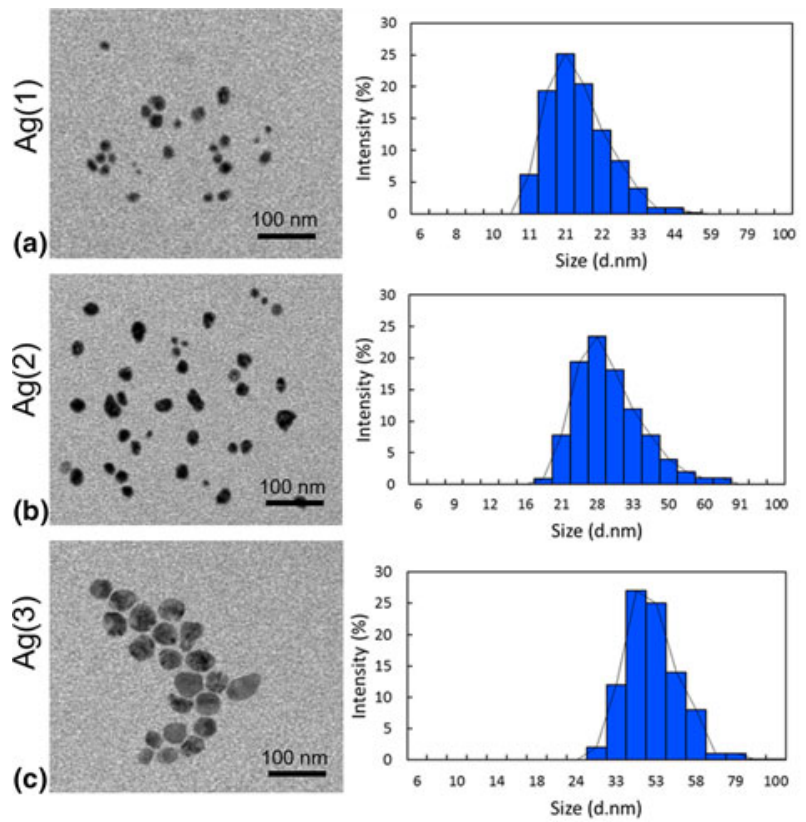

Figure 4: TEM images and size distributions of the synthesized particles at various UV light exposure time intervals (a) $1 \mathrm{~h}$, (b) $2 \mathrm{~h}$, and (c) $3 \mathrm{~h}$.

between AgNPs and PLA by attenuated total reflection (ATR)-Fourier transform infrared (FTIR) analysis. To achieve this, 3D-printed PLA scaffolds were immersed in silver precursor solution and then put in the UV irradiation chamber for 1 , 2 , and $3 \mathrm{~h}$. These samples were named as PLA/Ag(1), PLA/Ag (2), and PLA/Ag(3), respectively [Fig. 3(b)]. Zeta sizer and TEM analysis results showed that $\operatorname{Ag}(3)$ solutions have the highest PDI and great particle dispersity compared with $\mathrm{Ag}$ (2) and $\operatorname{Ag}(1)$ solutions (Table 2). In this regard, PLA/Ag(3) scaffolds were chosen to investigate the structural and thermal properties of AgNPs and 3D-printed PLA scaffolds.

\section{Structural properties of 3D-printed PLA and PLA/ Ag(3) scaffolds}

FTIR spectra of 3D-printed PLA and PLA/Ag(3) scaffolds are shown in Fig. 5. FTIR analysis was carried out to clarify whether there was an interaction between 3D-printed PLA scaffolds and in situ synthesized AgNPs. 3D-printed PLA indicated a characteristic absorption peak region between 2900 and $2851 \mathrm{~cm}^{-1}$ corresponding to the symmetric and asymmetric stretching vibration of the $\mathrm{CH}$ in $-\mathrm{CH}_{3}$. The most important
TABLE 2: Composition and key properties of the scaffolds

\begin{tabular}{lll}
\hline \hline Sample code & $\mathrm{AgNO}_{3}(\mathrm{w} / \mathrm{v})$ & UV irradiation time $(\mathrm{h})$ \\
\hline PLA (filament) & - & - \\
3D-printed PLA & - & - \\
PLA/Ag(1) & 0.5 & 1 \\
PLA/Ag(2) & 0.5 & 2 \\
PLA/Ag(3) & 0.5 & 3 \\
\hline \hline
\end{tabular}

band observed at $1747 \mathrm{~cm}^{-1}$ corresponding to the $\mathrm{C}=\mathrm{O}$ stretching from the ester group in 3D-printed PLA. The most significant difference between 3D-printed PLA scaffolds and in situ synthesized 3D-printed PLA scaffolds (PLA/Ag(3)) was the appearance of the $-\mathrm{OH}$ band, suggesting possible interactions between the hydroxyl group of PLA and in situ synthesized AgNPs. The results demonstrated that the broad-OH band's presence might be due to the van der Waals and electrostatic interactions between the partial positive charge on nanosilver and hydroxyl groups in PLA [31, 32]. The carbonyl peak was shifted from 1747 to $1752 \mathrm{~cm}^{-1}$ in PLA/Ag(3) compared with 3D-printed PLA. These results also proved that AgNPs interacted with carbonyl groups in PLA [31]. Overall, FTIR analysis results demonstrated that AgNPs were synthesized and adsorbed on 3D-printed PLA scaffolds by electrostatic or van der Waals forces.

\section{Thermal properties of 3D-printed PLA scaffolds following the FDM-based 3D printing process}

Thermal properties of PLA polymer have great importance from the $3 \mathrm{D}$ printing processing point of view. It has been reported that parameter variations in the FDM 3D printing process can change the polymer structure of PLA [33, 34, 35]. Thermal analysis was carried out to determine thermal transitions (crystallinity, glass transition, and melting temperatures) of PLA raw filament and 3D-printed PLA to investigate the changes after FDM printing.

Figure 6(a) indicates the measured differential scanning calorimetry (DSC) curves for various materials, including PLA filament, 3D-printed PLA, and PLA/Ag(3) scaffolds. The spectra summarized glass transition $\left(T_{\mathrm{g}}\right)$, crystallization $\left(T_{\mathrm{c}}\right)$, and melting temperatures $\left(T_{\mathrm{m}}\right)$. All samples represented endothermic peaks at around $170{ }^{\circ} \mathrm{C}$, associated with $T_{\mathrm{m}}$ of the PLA. Another endothermic peak was collected at around $60{ }^{\circ} \mathrm{C}$ for all samples, corresponding to $T_{\mathrm{g}}$. The thermal

TABLE 1: Characteristics of synthesized AgNPs.

\begin{tabular}{lcccccc}
\hline \hline AgNP solutions & $\mathrm{AgNO}_{3}(\mathrm{w} / \mathrm{v})$ & UV irradiation time $(\mathrm{h})$ & Hydrodynamic size $(\mathrm{nm})$ & Polydispersity index (PDI) & $\lambda_{\max }(\mathrm{nm})(\mathrm{SPR})$ & Zeta potential (mV) \\
\hline $\mathrm{Ag}(1)$ & 0.5 & 1 & $20 \pm 2.2$ & 0.28 & 422 \\
$\mathrm{Ag}(2)$ & 0.5 & 2 & $30 \pm 3.6$ & 0.21 & 4.68 \\
$\mathrm{Ag}(3)$ & 0.5 & 2 & $50 \pm 4.8$ & 0.19 & 4.37 & 435 \\
\hline \hline
\end{tabular}




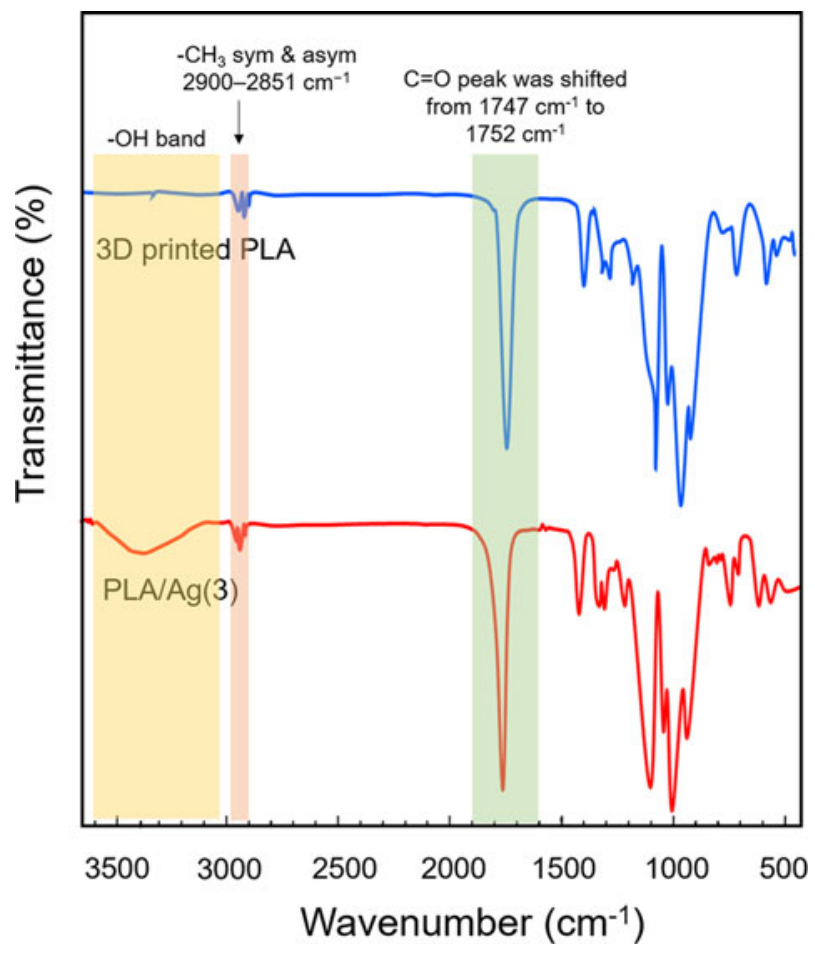

Figure 5: FTIR spectra of 3D-printed PLA and PLA/Ag(3) scaffolds.

peaks are consistent with the thermal properties of PLA reported by other researchers $[35,38]$. During the first heating run, PLA filament, 3D-printed PLA, and PLA/Ag(3) samples showed a sharp exothermic peak at around $98^{\circ} \mathrm{C}$. These peaks are attributed to the crystallization of samples along with the first heating run $\left(T_{\mathrm{c}}\right)$. Among them, 3D-printed PLA and PLA/Ag(3) samples were represented the sharper peaks compared with the PLA filament. These results can be attributed to the increased crystallinity of PLA after 3D printing. In this study, the scaffolds were left on the hot plate throughout the cooling process to keep their geometry intact and the cooling process took about $30 \mathrm{~min}$. Thus, at the end of the cooling process, the 3D-printed scaffolds cooled slowly and different variations occurred between the crystalline layers. These exothermic peaks are associated with the slow cooling of the 3D-printed scaffolds, fabricated using layer-by-layer deposition on hot plates $\left(65^{\circ} \mathrm{C}\right) 3 \mathrm{D}$ printer $[35,39,40]$. These results indicated that the FDM-based $3 \mathrm{D}$ printing process resulted in variations in the distribution of the polymer crystalline phases. This result showed that in situ AgNP synthesis and the presence of the AgNPs on 3D-printed scaffolds do not affect the PLA polymer structure. In the second heating run, sharp $T_{\mathrm{c}}$ exothermic peaks were decreased and form wide $T_{\mathrm{c}}$ peak from $80^{\circ} \mathrm{C}$ to $100{ }^{\circ} \mathrm{C}$. In the second heating run, $T_{\mathrm{m}}$ temperature of the PLA/ $\operatorname{Ag}(3)$ scaffolds slightly increases by $1-3{ }^{\circ} \mathrm{C}$ compared with the PLA filament, and 3D-printed PLA due to AgNP and PLA polymer matrix interactions. This result showed that in the first heating run, PLA polymer was melted and AgNPs are formed on the PLA surface blended with the PLA polymer matrix in PLA/Ag(3) scaffolds. Increased $T_{m}$ $\left(1-3{ }^{\circ} \mathrm{C}\right)$ for PLA/ $\operatorname{Ag}(3)$ scaffolds can be attributed to a blend of AgNP and PLA polymer matrix. In conclusion, in situ AgNP synthesis and the presence of the AgNPs on 3D-printed scaffolds do not affect the PLA polymer structure.
Figure 6: DSC thermal analysis and contact angle values for 3D-printed PLA and PLA/Ag scaffolds after different UV irradiation times. (a) DSC thermograms of the first heating run and the second heating run. (b) Ultrapure water droplets on scaffolds, and (c) contact angle measurements of the 3D-printed PLA scaffolds $\left({ }^{*} p<\right.$ 0.05 when compared with the PLA group, error bars represent $\mathrm{SD}$, with $n=3$ ).
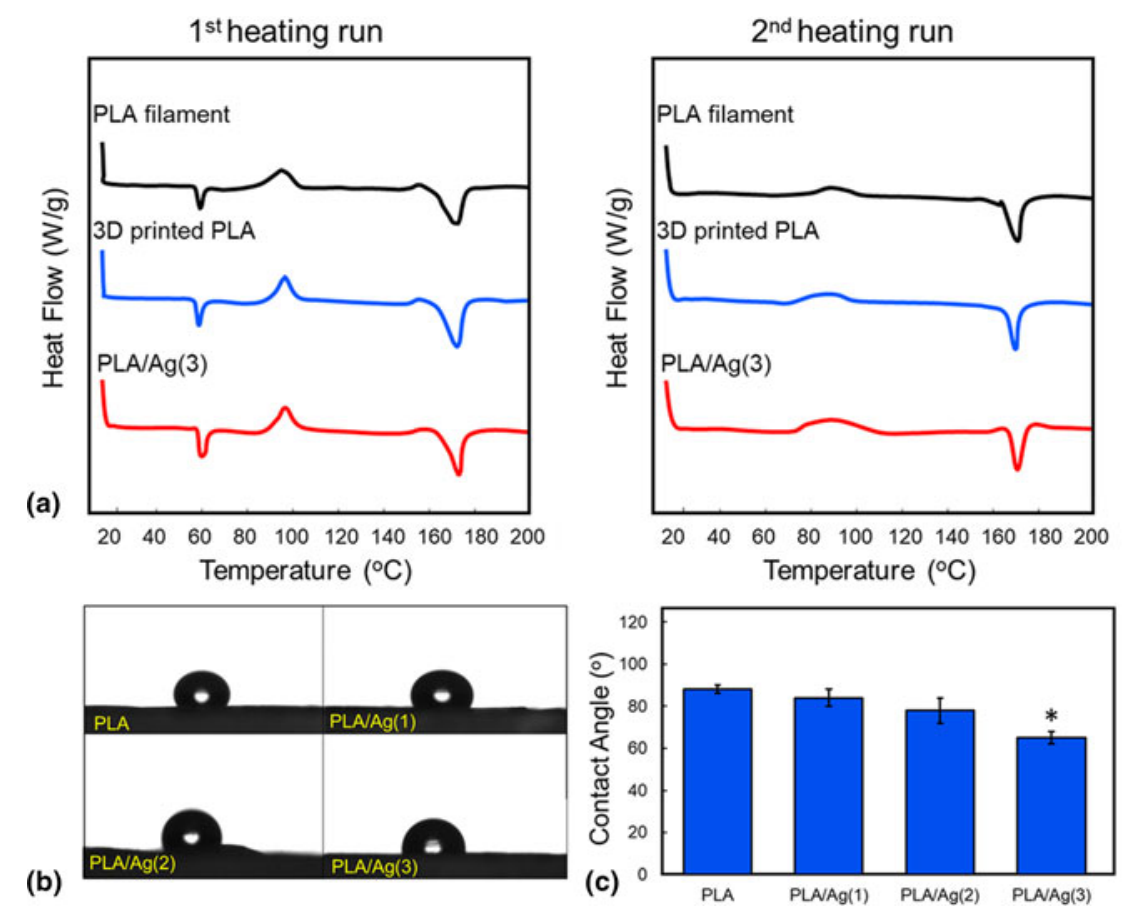
However, in the second run of the DSC analysis, the blend of AgNP and PLA might be induced a little change on the thermal properties of the PLA/Ag(3) scaffolds.

\section{Surface wettability}

The contact angle measurements represent the hydrophilicity, which mainly affects cell adhesion and protein adsorption on the 3D-printed PLA samples. Figures 6(b) and 6(c) demonstrate the results of water contact angle (WCA) measurements of 3D-printed PLA and in situ AgNP-synthesized 3D-printed PLA scaffolds. In the literature, PLA has known as a hydrophobic material. It has been reported that electrospun PLA nanofibers have a WCA of $149.5^{\circ}$ [41, 42]. The wettability results indicated that the 3D-printed PLA scaffolds (AgNP-free) showed a contact angle of $88.2^{\circ}$, which showed hydrophobic properties. UV-irradiated AgNP formation on 3D-printed PLA scaffolds dramatically changed the water droplet behavior on the PLA/Ag samples (Fig. 6(b)). After in situ AgNP synthesis, the WCA decreased to $84^{\circ}, 78^{\circ}$, and $65^{\circ}$ for PLA/Ag(1), $\mathrm{PLA} / \operatorname{Ag}(2)$, and $\mathrm{PLA} / \operatorname{Ag}(3)$, respectively. In a study, Wu et al. produced $3 \mathrm{D}$-printed PLA, which was functionalized by a polydopamine interlayer to firmly bond the halloysite nanotubes (HNTs) on the surfaces of the PLA pattern for guiding cell orientation. The WCA results demonstrated that the HNTs coating reduced the WCA of the 3D-printed PLA as low as $33.3 \pm 0.70^{\circ}$ [42]. In another study, Martin et al. designed 3D-printed PLA scaffolds with bioinspired citratehydroxyapatite nanoparticle surface coatings to provide closer structural support to native bone architecture. The results demonstrated that PLA scaffold had a hydrophilic surface with a WCA of $45 \pm 2^{\circ}$, while the WCA values of citratehydroxyapatite nanoparticle-functionalized PLA structure was of $17 \pm 4^{\circ}$ [43]. Rasoulianboroujeni et al. demonstrated a model 3D-printed scaffold, which is composed of poly (lactic-co-glycolic acid) $/ \mathrm{TiO}_{2}$ composite for bone tissue engineering applications. The addition of $\mathrm{TiO}_{2}$ nanoparticles increased the mechanical properties of scaffolds. The addition of $\mathrm{TiO}_{2}$ improved the hydrophilicity of the scaffolds by reducing the WCA from $90.5 \pm 3.2^{\circ}$ to $79.8 \pm 2.4^{\circ}$, which is in favor of cellular attachment and activity [44].

Basri et al. produced the antibacterial polyethersulfone/Ag nanocomposite materials and reported that AgNP significantly improved the membrane performance with increasing antibacterial activity and also the contact angle values decreased with an increase of AgNP loading [45]. In addition, Sile-Yuksel et al. showed that the WCA of polyethersulfone membranes decreased when increasing an AgNP ratio. It has been reported that the contact angle of polyethersulfone decreased from $71 \pm$ $4^{\circ}$ to $22 \pm 3^{\circ}$ when AgNP was introduced into polyethersulfone membranes [46]. Kasraei and Azarsina reported that functionalization of the composite resin with $\mathrm{AgNP}$, which has $50 \mathrm{~nm}$ particle size, resulted in consequently decreased the WCA. The authors suggested that the increased surface energy of composite resin may lead to lower contact angle measurements [47]. From the literature point of view, the WCA depends on nanoparticle size and nanoparticle concentration on the surface. Overall, these results were found in accordance with the literature.

\section{Conclusion}

This study was performed as an initial step for the in situ AgNP synthesis on the 3D-printed PLA scaffolds as a surface functionalization. The morphological characterization of AgNPs demonstrated that AgNPs were highly monodisperse with the particle size of $20 \pm 2.2,30 \pm 3.6$, and $50 \pm 4.8 \mathrm{~nm}$ under various UV light exposure times. The main advantage of the UV irradiation method over the conventional chemical methods is fast silver nanoparticle nucleation and generation on biomaterials. TEM, FTIR-ATR, and DSC analysis confirmed that AgNPs were in situ synthesized on 3D-printed PLA scaffolds using a facile UV irradiation method. Also, wettability analysis results showed that in situ synthesis of AgNPs on 3D-printed PLA scaffolds was significantly changed the surface hydrophilicity. In conclusion, this study provides a nontoxic, energy-efficient, and cost-effective processing methodology to produce AgNP-coated 3D-printed PLA scaffolds, which show promising results for biomedical applications including cell culture scaffolds, biosensors, and wound healing applications.

\section{Methodology}

\section{Design and fabrication of 3D-printed PLA scaffolds}

Ultimaker 2+ (Ultimaker B.V the Netherlands) 3D printer and Ultimaker Cura 3.6 software were used to fabricate 3D-printed PLA scaffolds ( $1 \mathrm{~cm}$ diameter). A biodegradable and biocompatible PLA filament with a diameter of $2.85 \mathrm{~mm}$ was purchased form Form Futura (the Netherlands). 3D-printed scaffolds were designed using the Sketchup modeling program and saved as stereolithography (.stl) file. Then, the final stl file was transferred to SD card to dictate the scaffold dimensions to the printer. The key parameters of the printing were set as an extruder temperature of $220^{\circ} \mathrm{C}$ and an extrusion print speed of $90 \mathrm{~mm} / \mathrm{s}$ and $0.4 \mathrm{~mm}$ layer height. The PLA filament was extruded through a circular nozzle with a diameter of $0.4 \mathrm{~mm}$.

For this study, three orthogonal scaffolds were designed with a layer orientation of $90^{\circ}$, and strut spaces were determined as $0.5,0.3$, and $0.2 \mathrm{~mm}$ for PLA 90-1, PLA 90-2, and PLA 90-3 scaffolds, respectively. Additionally, one tetragonal scaffold was designed with a layer orientation of $120^{\circ}$ (PLA 120). The printer extruder was deposited melted PLA layer 
by layer with the desired pattern. Before the $3 \mathrm{D}$ printing process, the platform was covered by heat-resistant tape, and during the $3 \mathrm{D}$ printing process, all materials were handled with gloved hands to minimize the contamination of the 3D-printed scaffolds. Bright-field images of the light microscope were employed to investigate the morphology of 3D-printed PLA scaffolds with orthogonal and tetragonal layer orientations.

\section{In situ synthesis of AgNPs by using the UV irradiation method}

For the in situ synthesis of AgNPs on 3D-printed PLA scaffolds, the silver precursor solution was prepared according to our previous reports $[13,14]$. In this method, $\mathrm{AgNO}_{3}$ was used as a silver precursor. Silver precursor stock solution was prepared by solubilizing the $0.5 \%(\mathrm{w} / \mathrm{v}) \mathrm{AgNO}_{3}$ in ultrapure water. Then, the silver precursor solution was vigorously stirred under constant stirring for $1 \mathrm{~h}$ at room temperature.

The size distribution and zeta potential analyzes were carried out to investigate the key properties of colloidal AgNPs in ultrapure water. To synthesize in situ AgNPs, the silver precursor solutions were irradiated utilizing a UV chamber with a UV lamp at $\lambda$ : $365 \mathrm{~nm}$ under constant stirring. UV light exposures were applied during 1, 2, and $3 \mathrm{~h}$ at room temperature. After the characterization of AgNPs in colloidal solutions, in situ AgNP synthesis was carried out on 3D-printed PLA scaffolds. For this purpose, 3D-printed PLA scaffolds were immersed in silver precursor solution and incubated during $1 \mathrm{~h}$ at a slow stirring rate. Then, 3D-printed PLA scaffolds were taken from solution and put in the UV irradiation chamber during 1,2 , and $3 \mathrm{~h}$. These samples were defined as PLA/Ag(1), PLA/Ag(2), and PLA/Ag(3), respectively (Table 1). Finally, AgNPs containing 3D-printed PLA scaffolds were washed three times with ultrapure water to remove unreacted $\mathrm{Ag}^{+}$ ions and kept at $40{ }^{\circ} \mathrm{C}$ in a vacuum oven overnight. The composition of $3 \mathrm{D}$-printed PLA scaffolds is given in Table 2.

\section{Characterization of AgNPs}

UV-visible spectroscopy was utilized to investigate the formation of AgNP in the first step. The absorbance of AgNPs in water was characterized by Shimadzu (UV-2600) UV-Vis spectrophotometers. The absorbance spectra were collected in the range of $300-800 \mathrm{~nm}$. Ultrapure water was used as a reference sample, and the measurements were taken after 1,2 , and $3 \mathrm{~h}$ UV irradiation of the samples. The hydrodynamic particle size and zeta potential analysis were carried out by using a Zetasizer Nano ZS analyzer (Malvern Instrument Ltd, Malvern, Worcestershire, UK). All experiments were triplicated $(n=3)$. Also, TEM was utilized to investigate size and geometry [37].

\section{Characterization of 3D-Printed PLA Scaffolds}

\section{ATR-FTIR spectroscopy}

Surface functional groups of PLA and in situ AgNP synthesis on 3D-printed PLA scaffolds were investigated by FTIR using the ATR attachment. The spectra were collected over the range of $3500-300 \mathrm{~cm}^{-1}$. The SpectraGryph software was used to normalize FTIR data.

\section{Differential scanning calorimetry}

DSC analysis was carried out using a TA Instruments Universal T4 5A to analyze the thermal transition of PLA filament, 3D-printed PLA and PLA/Ag(3). For this purpose, 5-10 mg of samples were sealed in aluminum pans and heated at a rate of $10^{\circ} \mathrm{C} / \mathrm{min}$ from $25^{\circ} \mathrm{C}$ to $200{ }^{\circ} \mathrm{C}$ (first heating run). Then, samples were cooled to $25^{\circ} \mathrm{C}$. After the cooling process, samples were re-heated from $25^{\circ} \mathrm{C}$ to $200^{\circ} \mathrm{C}$ at the same heating rate (second heating run). Glass transition $\left(T_{\mathrm{g}}\right)$, crystallization $\left(T_{\mathrm{c}}\right)$, and melting temperatures $\left(T_{\mathrm{m}}\right)$ were determined following the FDM-based $3 \mathrm{D}$ printing process.

\section{Wettability analysis}

The wettability of the $3 \mathrm{D}$-printed samples was determined by measuring the contact angles of ultrapure water on 3D-printed PLA and PLA/Ag(3) composite scaffolds with a contact angle measuring system (Attension Theta). To achieve this, 3D-printed scaffolds were attached on a microscope slide by using double-sided adhesive tape. The WCA values were determined with the angle at which the ultrapure water $(5 \mu \mathrm{L})$ interface met the 3D-printed scaffolds. The mean value of the three measurements was determined as the contact angle of each sample. The measurements were performed after droplet stabilization (20 s) [47]. All measurements were carried out at room temperature.

\section{Data analysis}

The results were analyzed using the GraphPad Prism software with one-way ANOVA. The statistical significance was set at $0.05(P<0.05)$. The standard deviation value was given as the standard error of the mean values.

\section{Acknowledgment}

S.C. thanks Elif Sarigol Calamak for production of 3Dprinted PLA scaffolds and acknowledges Hacettepe University, Faculty of Pharmacy, Department of Pharmaceutical Chemistry, for helping ATR-FTIR measurement of 3D-printed PLA scaffolds. M.E. acknowledges Amfibi Technical llc. for donating the Ultimaker $2+$ for this research. 


\section{References}

1. N. Dasgupta and C. Ramalingam: Silver nanoparticle antimicrobial activity explained by membrane rupture and reactive oxygen generation. Environ. Chem. Lett. 14, 477-485 (2016).

2. M. Sabela, S. Balme, M. Bechelany, J.M. Janot, and K. Bisetty: A review of gold and silver nanoparticle-based colorimetric sensing assays. Adv. Eng. Mater. 19, 1700270 (2017).

3. J.L. Elechiguerra, J.L. Burt, J.R. Morones, A. Camacho-Bragado, X. Gao, H.H. Lara, and M.J. Yacaman: Interaction of silver nanoparticles with HIV-1. J. Nanobiotechnol. 3, 6 (2005).

4. G. Liao, Q. Li, W. Zhao, Q. Pang, H. Gao, and Z. Xu: In-situ construction of novel silver nanoparticle decorated polymeric spheres as highly active and stable catalysts for reduction of methylene blue dye. Appl. Catal. A 549, 102-111 (2018).

5. K. Gudikandula and S. Charya Maringanti: Synthesis of silver nanoparticles by chemical and biological methods and their antimicrobial properties. J. Exp. Nanosci. 11, 714-721 (2016).

6. A. Barbasz, M. Oćwieja, and M. Roman: Toxicity of silver nanoparticles towards tumoral human cell lines U-937 and HL-60. Colloids Surf. B 156, 397-404 (2017).

7. V. Gurusamy, R. Krishnamoorthy, B. Gopal, and

V. Veeraravagan: Systematic investigation on hydrazine hydrate assisted reduction of silver nanoparticles and its antibacterial properties. Inorg. Nano-Met. Chem. 47, 761-767 (2017).

8. S. Huang, L. Zhou, M.-C. Li, Q. Wu, Y. Kojima, and D. Zhou: Preparation and properties of electrospun poly (vinyl pyrrolidone)/cellulose nanocrystal/silver nanoparticle composite fibers. Materials 9, 523 (2016).

9. P. Wagener, S. Ibrahimkutty, A. Menzel, A. Plech, and S. Barcikowski: Dynamics of silver nanoparticle formation and agglomeration inside the cavitation bubble after pulsed laser ablation in liquid. Phys. Chem. Chem. Phys. 15, 3068-3074 (2013).

10. H. Peng, A. Yang, and J. Xiong: Green, microwave-assisted synthesis of silver nanoparticles using bamboo hemicelluloses and glucose in an aqueous medium. Carbohydr. Polym.91, 348-355 (2013).

11. I.A. Wani, A. Ganguly, J. Ahmed, and T. Ahmad: Silver nanoparticles: Ultrasonic wave assisted synthesis, optical characterization and surface area studies. Mater. Lett. 65, 520-522 (2011).

12. Y. Liu, S. Chen, L. Zhong, and G. Wu: Preparation of high-stable silver nanoparticle dispersion by using sodium alginate as a stabilizer under gamma radiation. Radiat. Phys. Chem. 78, 251-255 (2009).

13. S. Calamak, E.A. Aksoy, C. Erdogdu, M. Sagıroglu, and K. Ulubayram: Silver nanoparticle containing silk fibroin bionanotextiles. J. Nanopart. Res. 17, 87 (2015).

14. S. Calamak, E.A. Aksoy, N. Ertas, C. Erdogdu, M. Sagıroglu, and K. Ulubayram: Ag/silk fibroin nanofibers: Effect of fibroin morphology on $\mathrm{Ag}^{+}$release and antibacterial activity. Eur. Polym. J. 67, 99-112 (2015).

15. A. Radon and D. Lukowiec: Silver nanoparticles synthesized by UV-irradiation method using chloramine $\mathrm{T}$ as modifier: Structure, formation mechanism and catalytic activity. CrystEngComm 20, 7130-7136 (2018).

16. K. Shameli, M.B. Ahmad, W.M.Z.W. Yunus, A. Rustaiyan, N.A. Ibrahim, M. Zargar, and Y. Abdollahi: Green synthesis of silver/montmorillonite/chitosan bionanocomposites using the UV irradiation method and evaluation of antibacterial activity. Int. J. Nanomed. 5, 875 (2010).

17. W.K. Son, J.H. Youk, and W.H. Park: Antimicrobial cellulose acetate nanofibers containing silver nanoparticles. Carbohydr. Polym. 65, 430-434 (2006).

18. A.M. Silva, C.B. de Araújo, S. Santos-Silva, and A. Galembeck: Silver nanoparticle in situ growth within crosslinked poly (ester-co-styrene) induced by UV irradiation: Aggregation control with exposure time. J. Phys. Chem. Solids 68, 729-733 (2007).

19. R. Patakfalvi, A. Oszko, and I. Dékány: Synthesis and characterization of silver nanoparticle/kaolinite composites. Colloids Surf. A 220, 45-54 (2003).

20. L.C. Courrol, F.R. de Oliveira Silva, and L. Gomes: A simple method to synthesize silver nanoparticles by photo-reduction. Colloids Surf. A 305, 54-57 (2007).

21. F. Kim, J.H. Song, and P. Yang: Photochemical synthesis of gold nanorods. J. Am. Chem. Soc. 124, 14316-14317 (2002).

22. X. Chen, C. Gao, J. Jiang, Y. Wu, P. Zhu, and G. Chen: 3D printed porous PLA/nHA composite scaffolds with enhanced osteogenesis and osteoconductivity in vivo for bone regeneration. Biomed. Mater. 14, 065003 (2019).

23. B.N. Teixeira, P. Aprile, R.H. Mendonça, D.J. Kelly, and R.M.D.S.M. Thiré: Evaluation of bone marrow stem cell response to PLA scaffolds manufactured by $3 \mathrm{D}$ printing and coated with polydopamine and type I collagen. J. Biomed. Mater. Res. B 107, 37-49 (2019).

24. E. Sarigol-Calamak and C. Hascicek: Tissue scaffolds as a local drug delivery system for bone regeneration. In Cutting-Edge Enabling Technologies for Regenerative Medicine, H.J. Chun, C.H. Park, I.K. Kwon and G. Khang, eds. (Springer, Singapore, 2018), pp. 475-493.

25. W.J. Choi, K.S. Hwang, H.J. Kwon, C. Lee, C.H. Kim, T.H. Kim S.W. Heo, J.-H. Kim, and J.-Y. Lee: Rapid development of dual porous poly (lactic acid) foam using fused deposition modeling (FDM) 3D printing for medical scaffold application. Mater. Sci. Eng. C 110693 (2020).

26. C.-T. Kao, C.-C. Lin, Y.-W. Chen, C.-H. Yeh, H.-Y. Fang, and M.-Y. Shie: Poly (dopamine) coating of 3D printed poly (lactic acid) scaffolds for bone tissue engineering. Mater. Sci. Eng. C 56, 165-173 (2015). 
27. X. Li, Y. Wang, M. Guo, Z. Wang, N. Shao, P. Zhang, X. Chen, and Y. Huang: Degradable three dimensional-printed polylactic acid scaffold with long-term antibacterial activity. ACS Sustain. Chem. Eng. 6, 2047-2054 (2018).

28. R. Scaffaro, F. Lopresti, A. Sutera, L. Botta, R.M. Fontana, and G. Gallo: Plasma modified PLA electrospun membranes for actinorhodin production intensification in Streptomyces coelicolor immobilized-cell cultivations. Colloids Surf. B 157, 233-241 (2017).

29. Y. Wang, R. Ren, J. Ling, W. Sun, and Z. Shen: One-pot "grafting-from" synthesis of amphiphilic bottlebrush block copolymers containing PLA and PVP side chains via tandem ROP and RAFT polymerization. Polymer 138, 378-386 (2018).

30. R.A. Quirk, W.C. Chan, M.C. Davies, S.J. Tendler, and

K.M. Shakesheff: Poly (L-lysine)-GRGDS as a biomimetic surface modifier for poly (lactic acid). Biomaterials 22, 865-872 (2001).

31. S. Alipilakkotte and L. Sreejith: Green synthesized PLA/silver nanoparticle probe for sensing of hydrogen peroxide in biological samples. Mater. Lett. 217, 33-38 (2018).

32. X. Xu, Q. Yang, Y. Wang, H. Yu, X. Chen, and X. Jing: Biodegradable electrospun poly (L-lactide) fibers containing antibacterial silver nanoparticles. Eur. Polym. J. 42, 2081-2087 (2006).

33. M.A. Cuiffo, J. Snyder, A.M. Elliott, N. Romero, S. Kannan, and G.P. Halada: Impact of the fused deposition (FDM) printing process on polylactic acid (PLA) chemistry and structure. Appl. Sci. 7, 579 (2017).

34. G. Spinelli, R. Kotsilkova, E. Ivanov, I. Petrova-Doycheva, D. Menseidov, V. Georgiev, R. Di Maio, and C. Silvestre: Effects of filament extrusion, 3D printing and hot-pressing on electrical and tensile properties of poly (lactic) acid composites filled with carbon nanotubes and graphene. Nanomaterials 10, 35 (2020).

35. Y. Song, Y. Li, W. Song, K. Yee, K.-Y. Lee, and V.L. Tagarielli: Measurements of the mechanical response of unidirectional 3D-printed PLA. Mater. Des. 123, 154-164 (2017).

36. G.-N. Xu, X.-L. Qiao, X.-L. Qiu, and J.-G. Chen: Preparation and characterization of stable monodisperse silver nanoparticles via photoreduction. Colloids Surf. A 320, 222-226 (2008).

37. S. Calamak and K. Ulubayram: Polyethylenimine-mediated gold nanoparticle arrays with tunable electric field enhancement for plasmonic applications. J. Mater. Sci. Mater. Electron. 30, 10013-10023 (2019).
38. Y.-M. Corre, A. Maazouz, J. Duchet, and J. Reignier: Batch foaming of chain extended PLA with supercritical $\mathrm{CO}_{2}$ : Influence of the rheological properties and the process parameters on the cellular structure. J. Supercrit. Fluids 58, 177-188 (2011).

39. J. Guillen and W. Cantwell: The influence of cooling rate on the fracture properties of a thermoplastic-based fibre-metal laminate. J. Reinf. Plast. Compos. 21, 749-772 (2002).

40. A. Jalali, M.A. Huneault, and S. Elkoun: Effect of thermal history on nucleation and crystallization of poly (lactic acid). J. Mater. Sci. 51, 7768-7779 (2016).

41. H. Liu, W. Li, B. Luo, X. Chen, W. Wen, and C. Zhou: Icariin immobilized electrospinning poly (L-lactide) fibrous membranes via polydopamine adhesive coating with enhanced cytocompatibility and osteogenic activity. Mater. Sci. Eng. C 79, 399-409 (2017).

42. F. Wu, J. Zheng, Z. Li, and M. Liu: Halloysite nanotubes coated 3D printed PLA pattern for guiding human mesenchymal stem cells (hMSCs) orientation. Chem. Eng. J. 359, 672-683 (2019).

43. V. Martin, I.A. Ribeiro, M.M. Alves, L. Gonçalves, R.A. Claudio, L. Grenho, M.H. Fernandes, P. Gomes, C.F. Santos, and A.F. Bettencourt: Engineering a multifunctional 3D-printed PLA-collagen-minocycline-nanohydroxyapatite scaffold with combined antimicrobial and osteogenic effects for bone regeneration. Mater. Sci. Eng. C 101, 15-26 (2019).

44. M. Rasoulianboroujeni, F. Fahimipour, P. Shah, K. Khoshroo, M. Tahriri, H. Eslami, A. Yadegari, E. Dashtimoghadam, and L. Tayebi: Development of 3D-printed $\mathrm{PLGA} / \mathrm{TiO}_{2}$ nanocomposite scaffolds for bone tissue engineering applications. Mater. Sci. Eng. C 96, 105-113 (2019).

45. H. Basri, A.F. Ismail, and M. Aziz: Polyethersulfone (PES)-silver composite UF membrane: Effect of silver loading and PVP molecular weight on membrane morphology and antibacterial activity. Desalination 273, 72-80 (2011).

46. M. Sile-Yuksel, B. Tas, D.Y. Koseoglu-Imer, and I. Koyuncu: Effect of silver nanoparticle (AgNP) location in nanocomposite membrane matrix fabricated with different polymer type on antibacterial mechanism. Desalination 347, 120-130 (2014).

47. S. Kasraei and M. Azarsina: Addition of silver nanoparticles reduces the wettability of methacrylate and silorane-based composites. Braz. Oral Res. 26, 505-510 (2012). 